\title{
Ultraschall in der Diagnostik von Polyneuropathien
} Ultrasonography in the Diagnosis of Polyneuropathies

Autor

Institut
N. Üçeyler

Neurologische Klinik, Universität Würzburg, Würzburg

\author{
Schlüsselwörter \\ - hochauflösender \\ Nervenultraschall \\ - Polyneuropathie \\ - Diagnostik \\ - Bildgebung \\ - Differenzialdiagnostik \\ Keywords \\ - high-resolution nerve \\ ultrasonography \\ - polyneuropathy \\ - diagnostics \\ - imaging technique \\ - differential diagnosis
}

Bibliografie

DOI http://dx.doi.org/

10.1055/s-0042-117504

Online-Publikation: 10.10.2016

Akt Neurol 2016; 43: 548-556

(c) Georg Thieme Verlag KG

Stuttgart · New York

ISSN 0302-4350

Korrespondenzadresse Priv.-Doz. Dr. Nurcan Üçeyler Neurologische Klinik Universität Würzburg Josef-Schneider-Straße 11 97080 Würzburg ueceyler_n@ukw.de

\section{Zusammenfassung}

$\checkmark$

Zur Diagnosestellung von Polyneuropathien (PNP) stehen neben der ausführlichen Anamneseerhebung und der klinisch-neurologischen Untersuchung in erster Linie die Elektroneurografie und Elektromyografie zur Verfügung. Der hochauflösende Nervenultraschall hat als weiteres Diagnostikum bei PNP in den letzten Jahren zunehmend an Bedeutung gewonnen. Diese nichtinvasive und nebenwirkungsfreie Untersuchungstechnik eröffnet zum klinischen Befund und den Ergebnissen der Nervenleitungsstudien eine weitere diagnostische Dimension. Der Nervenultraschall erlaubt die sonomorphologische Beurteilung der peripheren Nerven und ihrer Umgebung und ermöglicht dadurch u.a. die Erkennung von Differenzialdiagnosen. Insbesondere bei negativen oder nicht eindeutigen elektroneurografischen Untersuchungsergebnissen kann der Befund des Nervenultraschalls wertvolle Zusatzinformationen geben. Hinzu kommt, dass mittels Nervenultraschall auch Abschnitte des peripheren Nervensystems untersucht werden können, die die neurografische Diagnostik nicht oder nur eingeschränkt erreichen kann, wie etwa die Nervenplexus und Spinalwurzeln. Wenn nach ausführlicher Diagnostik eine Nervenbiopsie zur Klärung der PNP Ursache notwendig wird, kann der hochauflösende Ultraschall bei der Auswahl einer geeigneten Biopsiestelle sehr hilfreich sein und somit die diagnostische Treffsicherheit erhöhen. Die Datenlage zur diagnostischen Wertigkeit des hochauflösenden Nervenultraschalls bei PNP wird stetig besser, wobei auch die Herausforderungen immer klarer werden, die in den nächsten Jahren angegangen und gelöst werden müssen. Die standardisierte Untersuchung großer, prospektiv rekrutierter, sowie klinisch und elektrophysiologisch gut charakterisierter Patientengruppen unter weitest gehender Ausschaltung möglicher konfundierender Einflussfaktoren wird

\section{Abstract \\ $\nabla$}

The diagnosis of polyneuropathies (PNP) is made by a detailed patient history, a thorough neurological examination, and neurophysiological tests. In recent years, high-resolution nerve ultrasonography has increasingly gained importance as an additional diagnostic tool in PNP. This non-invasive technique with so far no known side effects opens a further diagnostic dimension to the clinical and electrophysiological assessment of patients with neuropathies. By visualizing the peripheral nerves and their surrounding structures, nerve sonography enables sonomorphological assessment that facilitates making differential diagnoses. Particularly in cases where neurophysiological tests give negative or ambiguous results, findings with high-resolution nerve sonography may give valuable additional diagnostic hints. Moreover, parts of the peripheral nervous system that can hardly be reached or are not at all reachable by electrophysiological methods such as the plexus or the spinal roots can easily be visualized and assessed using nerve sonography. If, after extensive diagnostics, a nerve biopsy becomes necessary, high-resolution nerve sonography can be very helpful in finding a suitable area for biopsy and thus increase diagnostic accuracy. Data on the diagnostic value of high-resolution nerve sonography in PNP is constantly improving; in parallel, the challenges that need to be addressed and solved in the coming years become clearer. Standardized examination of large, prospectively recruited, and clinically and electrophysiologically well characterized patient groups together with maximum control of potential influencing factors will substantially help to further anchor high-resolution nerve ultrasonography in the diagnostics of PNP. 
in den kommenden Jahren entscheidend dazu beitragen, den hochauflösenden Nervenultraschall bei der PNP-Diagnostik weiter zu verankern.

\section{Polyneuropathien \\ $\nabla$}

Polyneuropathien (PNP) sind eine heterogene Gruppe von Erkrankungen des peripheren Nervensystems, die alle seine Abschnitte von den dünn- und unbemarkten A-delta und C-Nervenfasern (den sogenannten „small fibers“) bis zu den Nervenwurzeln betreffen können. Je nach befallener Komponente kommt es isoliert oder in Kombination zu motorischen, sensiblen und/oder autonomen Symptomen mit oder ohne neuropathische Schmerzen. Die Beschwerden breiten sich typischerweise von distal nach proximal aus und beginnen meist an den Zehen und Füßen. PNP können nach unterschiedlichen Gesichtspunkten kategorisiert werden wie z. B. nach ihrer Dynamik (akut - subakut - chronisch), der befallenen Nervenkomponente (axonal - entmarkend - gemischt), dem klinischen Bild (distal - proximal - symmetrisch - asymmetrisch; motorisch - sensibel autonom) und der Ätiologie (erworben - hereditär - idiopathisch). Wichtig ist, dass die klinische Präsentation meist nur wenige Rückschlüsse auf die Ätiologie erlaubt, d.h. dass PNP verschiedener Genese das gleiche klinische Bild verursachen können. Daher hat die Zusatzdiagnostik bei der Ursachenabklärung der PNP große Bedeutung, zumal erworbene Neuropathien potenziell behandelbar sind.

\section{Diagnostik bei PNP \\ $\nabla$}

Die Hauptsäulen der PNP Diagnostik sind die ausführliche Anamnese, die vollständige klinisch-neurologische Untersuchung und die elektrophysiologische Untersuchung mittels Elektroneurografie und Elektromyografie. Die hieraus gewonnenen Informationen bilden die Basis für die erste Arbeitshypothese zur PNPUrsache und helfen, die Differenzialdiagnosen einzugrenzen. Insbesondere die neurophysiologischen Untersuchungen geben Aufschluss über das Ausmaß des Nervenbefalls, die Akuität und die befallenen Nervenkomponenten (motorisch - sensibel; axonal - entmarkend). Je nach Verdachtsdiagnose kommen dann weitere Laboruntersuchungen und die Liquoranalyse hinzu. Falls die PNP-Ursache unklar bleibt ist bei Verdacht auf eine entzündliche Neuropathie eine Nervenbiopsie zu erwägen, die üblicherweise von chirurgisch gut zugänglichen rein sensiblen Nerven entnommen werden kann (z.B. N. suralis, N. peronaeus superficialis, N. radialis superficialis). Eine Hauptfrage, die die invasive Nervenbiopsie beantwortet ist, ob es sich um eine inflammatorische PNP handelt, die mit immunsuppressiven bzw. immunmodulatorischen Medikamenten behandelbar wäre. Neben diesem klassischen diagnostischen Algorithmus wurden in den letzten Jahren die MR-Neurografie und der hochauflösende Nervenultraschall als zusätzliche bildgebende Verfahren entwickelt, die die Beurteilung der peripheren Nerven um wesentliche neue Dimensionen erweitern. Hinsichtlich der Entwicklung und Wertigkeit der MR-Bildgebung bei der Diagnostik von PNP wird auf entsprechende Literatur verwiesen $[1,2]$, während im Folgenden die Bedeutung des hochauflösenden Nervenultraschalls näher beleuchtet wird.

\section{Hochauflösender Ultraschall}

\section{$\nabla$}

Der Einsatz von Ultraschall hat sich in den letzten ca. 20 Jahren bei der Untersuchung der peripheren Nerven über den Plexus brachialis bis zu den zervikalen Nervenwurzeln immer weiter etabliert. Parallel zu dieser Entwicklung verbesserte sich auch die Leistungsfähigkeit der Ultraschallgeräte und ihrer Bildverarbeitungssoftware. Mittlerweile stehen hochauflösende Ultraschallsonden zur Verfügung, die mit einer durchschnittlichen Frequenzleistung von $12-20 \mathrm{MHz}$ und mehr eine so hohe Bildqualität erreichen, dass nicht nur großkalibrige Nerven bis zur Faszikelstruktur analysiert und beurteilt werden können, sondern auch feine sensible und motorische Nervenendäste. Die dynamische Untersuchung im Quer- und Längsschnitt liefert wertvolle Informationen zur Morphologie des peripheren Nervensystems und des umgebenden Gewebes. Es können u.a. eine Kaliberzunahme im Sinne einer generalisierten oder fokalen Vergrößerung des Nervenquerschnitts (cross sectional area, CSA), Veränderungen der Nervenechogenität (echoarm - echoreich), Faszikelgröße (normal - ödematös aufgetrieben), Kontinuitätsunterbrechungen, neurale und extraneurale Raumforderungen, sowie mittels der Dopplerfunktion die Nervenvaskularisation beurteilt werden. Unter den zahlreichen möglichen Messparametern bei der quantitativen Analyse peripherer Nerven hat sich die CSA als am zuverlässigsten durchgesetzt [3] und kann mithilfe der Gerätesoftware einfach ermittelt werden ( $\bullet$ Abb. 1). Der gesunde periphere Nerv lässt sich im Querschnitt als Honigwaben-artige, rundlich-ovale, echoreiche Struktur mit punktförmig erscheinenden echoarmen Faszikeln darstellen, während er im Längsschnitt mit seinem echoarmen Endoneurium und echoreichen Perineurium ähnlich wie ein elektrisches Kabel aussieht ( $\bullet$ Abb. 1). Domänen der Anwendung des Nervenultraschalls sind bspw. Kompressionssyndrome, traumatische Nervenläsionen, sowie Raumforderungen und Tumore; zudem ist die Ultraschallsteuerung bei verschiedenen Interventionen wie z. B. intramuskuläre Injektionen oder etwa in der Anästhesie sehr nützlich. Die systematische Untersuchung von PNP Patienten mittels Nervenultraschall entwickelte sich erst in den letzten Jahren.

\section{Hochauflösender Ultraschall bei PNP - die Datenlage $\nabla$}

Bei der Untersuchung von Patienten mit Neuropathien mittels hochauflösendem Ultraschall ist die Datenlage hinsichtlich Ergebnissen, die an großen, prospektiv rekrutierten und verblindet untersuchten Studien- und Kontrollkohorten erhoben wurden, zwar noch spärlich, verbessert sich aber zunehmend [4]. Während in früheren Studien überwiegend die Nerven der oberen Extremitäten untersucht wurden, weil sie sonografisch leichter zugänglich sind, werden in aktuellen Studien immer mehr auch die bei PNP grundsätzlich häufiger und stärker betroffenen Nerven der unteren Extremitäten, sowie die proximalen Abschnitte des peripheren Nervensystems, also Plexus und Nervenwurzeln analysiert [5-7]. Zu beachten ist allerdings, dass aufgrund weitgehenden Mangels an national und international bindenden Standards in den jeweiligen Studien die untersuchten Nerven, die gewählten Messorte und auch die berichteten sonografischen Parameter sehr verschieden sind, was den aufgrund zahlreicher weiterer Einflussfaktoren ohnehin nur eingeschränkt möglichen Datenvergleich zwischen den Studien zusätzlich erschwert [4]. 

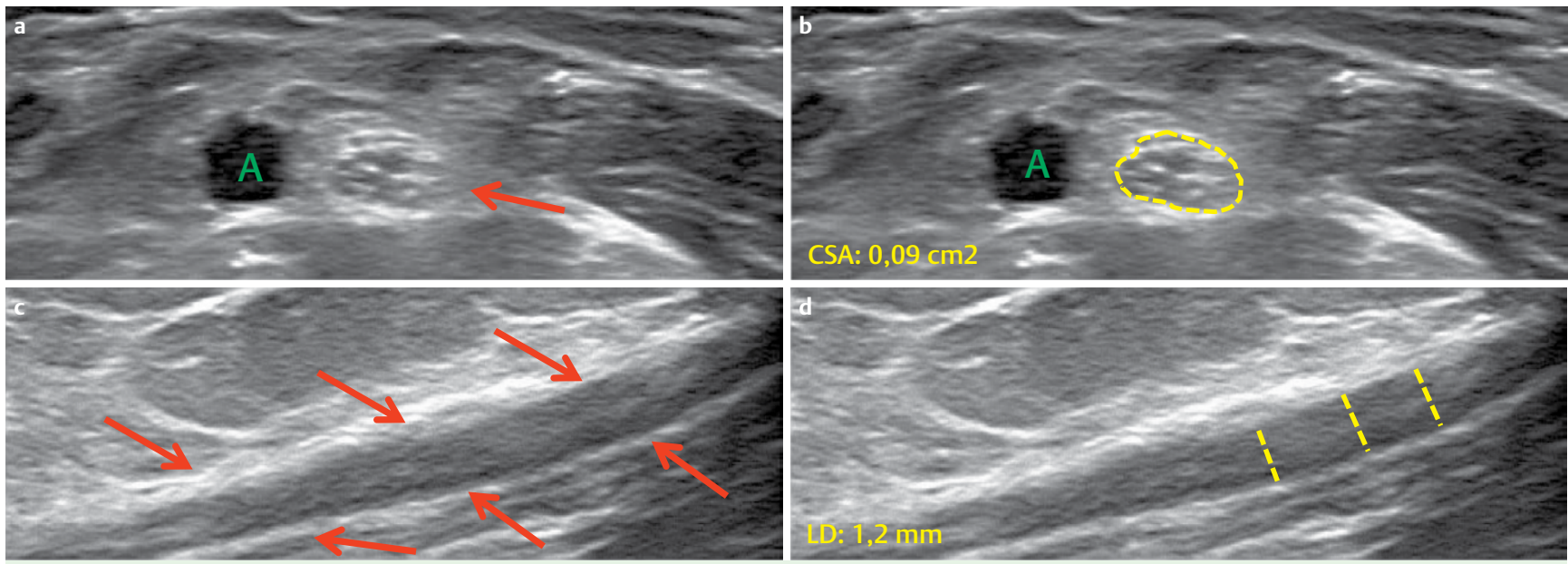

Abb. 1 Normaler N. medianus im Querschnitt in Höhe Ellenbeuge (a, b; grünes „A“ markiert die A. brachialis) und N. ulnaris im Längsschnitt in Höhe ca. Unterarmmitte $\mathbf{c}$, $\mathbf{d}$. In a und $\mathbf{c}$ weisen die roten Pfeile auf den jeweiligen Nerven. Im Querschnitt erscheint der gesunde Nerv a, b rundlich-oval und hat eine Honigwaben-artige Binnenstruktur mit echoreichem Endo- und Perineurium, sowie echoarmen, punktförmig erscheinenden Faszikeln. Im Längsschnitt wirkt der gesunde Nerv Kabel-artig mit echoreichem Perineurium und echoarmem Endoneurium. In a und $\mathbf{c}$ wird der jeweilige

\section{Mononeuropathien bei Engpasssyndromen}

Am längsten angewendet und daher am besten etabliert ist die Untersuchung mittels hochauflösendem Ultraschall bei Mononeuropathien im Rahmen von Kompressionssyndromen wie z.B. beim Karpaltunnelsyndrom, dem Cubitaltunnel-Syndrom oder bei der Kompression des N. peronaeus am Fibulaköpfchen. Da insbesondere die Untersuchung der Armnerven technisch einfach ist, hat sich der hochauflösende Nervenultraschall bei der Diagnostik dieser Engpasssyndrome in Ergänzung zur klinischen und elektrophysiologischen Zusatzdiagnostik sehr gut etabliert. Hauptbefund ist die Verdickung des jeweiligen Nerven proximal seines Eintauchens unter die natürliche Engstelle, während das Nervenkaliber an einer weiter proximalen bzw. distalen Stelle normal ist. Neben den morphologischen Eigenschaften im Sinne von deutlicher Kaliberzunahme und oft ödematöser Schwellung der Faszikel wird auch die Bestimmung einer CSA-Ratio empfohlen, bei der die CSA unmittelbar proximal des Engpasses mit derjenigen an einer von der Engpassstelle weiter proximal gelegenen Nervenstelle verglichen wird [8] (beispielhaft für den $\mathrm{N}$. medianus wäre die CSA-Ratio nach Hobson-Web und Kollegen als Quotient aus der Querschnittsfläche des N. medianus am Handgelenk und der Querschnittsfläche des Nerven ca. $12 \mathrm{~cm}$ proximal davon in etwa Mitte des Unterarms zu berechnen [9]). Da der Fokus dieser Übersichtsarbeit auf den PNP liegt wird für weitere Informationen zum Thema Ultraschall bei Kompressionssyndromen auf separate Literatur verwiesen [10].

\section{Hereditäre Neuropathien}

Bei der Untersuchung von Patienten mit hereditärer Neuropathie mit Neigung zu Druckläsionen (HNPP) wurden in Fallberichten [11-13], Studien mit [14,15] und ohne Kontrollgruppe [16-18] jeweils überwiegend verdickte Nerven der oberen $(\mathrm{N}$. medianus, N. ulnaris) und unteren Extremitäten (N. tibialis, N. peronaeus) beschrieben. Die CSA-Vergrößerungen wurden überwiegend an Engpassstellen gefunden ohne wesentliche Schwellung der Faszikel [18]. Auch bei der Untersuchung von Patienten mit einer Charcot-Marie-Tooth (CMT) Neuropathie (CM-

Nerv ohne Markierung gezeigt. In b ist die Markierung für die Bestimmung der cross sectional area (CSA) eingezeichnet (gelbe gestrichelte Linie), wobei die manuell eingefügte Kennzeichnungslinie definitionsgemäß innerhalb des echoreichen Epineuriums liegen muss. Die CSA selbst wird von der Gerätesoftware automatisch berechnet. In $\mathbf{d}$ sieht man die manuelle Markierung (gelbe gestrichelte Linien) zur Bestimmung des Längsdurchmessers (LD). Auch hierbei gilt, dass die Markierung innerhalb der echoreichen perineuralen Nervenränder liegen muss. CSA: cross sectional area.

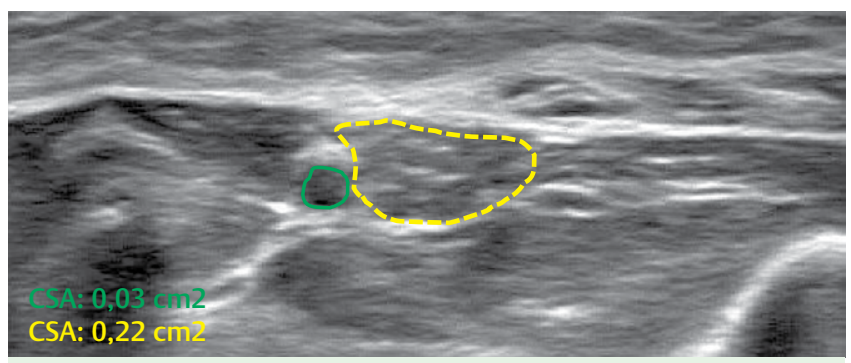

Abb. 2 Deutlich verdickter N. ulnaris am distalen Unterarm (a, gelbe gestrichelte Linie) in direkter Nachbarschaft zur A. ulnaris (mit grüner Linie umrandet). Während auf dieser Höhe der normale N. ulnaris etwa die gleiche Größe wie die benachbarte A. ulnaris hat, weist der Nerv in diesem Fall einer Patientin mit hereditärer Polyneuropathie (hereditäre motorische und sensible Neuropathie Typ 1, HMSN I) ein deutlich verdicktes Kaliber auf. CSA: cross sectional area.

T1A, CMT1B, CMT2, CMTX) wurden die sonografischen Befunde überwiegend an den Armnerven erhoben und zeigten bei demyelinisierenden Neuropathien eher eine generalisierte CSA-Vergrößerung ohne wesentliche Faszikelschwellung [15,19-23]; bei der axonalen CMT2 fand sich dagegen eine Vergrößerung der Faszikel ohne wesentliche Nervenschwellung [15]. In $\odot \mathbf{A b b} .2$ ist dies am Beispiel einer Patientin mit einer genetisch gesicherten hereditären motorischen und sensiblen Neuropathie (HMSN) Typ 1 demonstriert: während der N. ulnaris am distalen Unterarm normalerweise etwa so groß ist wie die begleitende A. ulnaris, ist er bei dieser Patientin um ein mehrfaches vergrößert und dies über seinen gesamten am Unter- und Oberarm einsehbaren Verlauf. Der sonologische Nachweis solcher generalisierter CSAVergrößerungen kann u.a. bei der differenzialdiagnostischen Abgrenzung einer erworbenen vs. hereditären Neuropathie wertvoll sein, da bei den erworbenen inflammatorischen PNP (s.u.) häufig fokale CSA-Vergrößerungen mit auch Ödem und Faszikelschwellung gefunden werden. Ob sich mittels hochauflösendem Ultraschall sogar Untergruppen hereditärer Neuropathien voneinander unterscheiden lassen [18] bzw. in wie fern 
der Einsatz von Score-Systemen die Differenzialdiagnostik erleichtern kann [24] bedarf weiterer Untersuchungen und Validierung an prospektiv rekrutierten, großen, multizentrischen und sehr gut definierten Patientensubgruppen. Bei den bisherigen Studien handelt es sich meist um kleine Fallzahlen z.T. ohne genetische Sicherung der Diagnose [25].

\section{Immunvermittelte Neuropathien}

Zusammenfassend handelt es sich bei den nervensonografischen Befunden bei Patienten mit immunvermittelten Neuropathien in erster Linie um Verdickungen des Nervenquerschnitts, die fokal oder generalisiert auftreten können. Ultraschalldaten zu Patienten mit einem Guillain-Barré-Syndrom (GBS) wurden in einem pädiatrischen Fallbericht [26] und einer weiteren Studie an erwachsenen Patienten [20] veröffentlicht. In diesen Arbeiten wurden überwiegend die Armnerven untersucht und es wurden fokale CSA-Vergrößerungen und ödematöse Schwellungen der Nervenfaszikel nachgewiesen. Neue Studien zeigen, dass auch der $\mathrm{N}$. vagus und insbesondere proximale Nervenabschnitte wie die Nervenwurzeln bereits in sehr frühen Stadien eines GBS durch eine deutliche Schwellung auffallen [5,27], die im Verlauf abnimmt [28]. Insbesondere der Nachweis einer Nervenwurzelverdickung kann bei einem beginnenden GBS und bei atypischer klinischer Präsentation oder elektrophysiologischen Befunden somit differenzialdiagnostisch nützlich sein.

Ähnlich sind die Befunde bei der chronischen inflammatorischen demyelinisierenden Polyneuropathie (CIDP), wobei die Ergebnisse und ihre Interpretationen hier nicht ganz so einheitlich sind. In Fallberichten [29-32] und Studien [20,33,34] wurden immer wieder fokale CSA-Vergrößerungen, Faszikelschwellungen und z.T. eine Hypervaskularisation an den überwiegend untersuchten Nerven der oberen Extremitäten beschrieben. Während in einer retrospektiven Analyse auch ein Zusammenhang mit der Erkrankungsaktivität und den sonografischen Befunden hergestellt werden konnte [35], fand sich in einer rezenten prospektiven Studie keine Korrelation mit den elektrophysiologischen Daten oder der funktionellen Einschränkung der Patienten [7]. Andere Autoren fanden zwar ebenfalls keine Korrelation mit den elektrophysiologischen, aber doch mit den klinischen Daten der untersuchten CIDP Patienten [36]. Im Gegensatz dazu wird in einer aktuellen Studie eine deutliche Korrelation der elektrophysiologischen Befunde der untersuchten CIDP Patienten mit den mittels Nervenultraschall erhobenen Daten berichtet [37], die in Anbetracht der Anzahl der untersuchten Nerven, des prospektiven Studiendesigns, sowie der Leistungs- fähigkeit der verwendeten Ultraschallsonde im Vergleich zu den bisherigen Fallberichten und kleinen Studien möglicherweise richtungsweisend ist. Die Frage, ob sich Befunde mittels hochauflösendem Nervenultraschall bei CIDP unter Therapie verändern wird in einer kürzlich veröffentlichten Studie beantwortet. Dabei wurden je 21 Patienten mit neu diagnostizierter bzw. länger bestehender CIDP mit Therapie seit > 6 Monaten untersucht. Es zeigte sich, dass die CSA mit zunehmender Krankheitsdauer zunimmt und positiv mit der Therapieverzögerung korrelieren [38]. In einer retrospektiven Studie wurde eine Normalisierung der CSA gefunden, wenn die Patienten mit klinischer und/oder elektrophysiologischer Verbesserung auch auf die Therapie angesprochen hatten [35]. Insgesamt wird das sonografische Muster bei CIDP in den verschiedenen Arbeiten uneinheitlich beschrieben [39]; auch ist noch nicht klar, ob und wenn ja, in wie weit die mittels Ultraschall erhobenen Befunde mit dem klinischen Bild, der Krankheitsprognose und den Befunden der neurophysiologischen Untersuchungen korrelieren. Weitere prospektive Studien an gut definierten großen Patientenkohorten im Vergleich mit gesunden Kontrollen sind notwendig, um die aktuell noch bestehenden Diskrepanzen zu klären. Zudem bleibt auch in diesem Fall abzuwarten, ob Score-Konstrukte $[40,41]$ sich bei der Differenzialdiagnostik etwa zwischen akuten und chronischen Neuropathien bzw. Subgruppen von immunvermittelten Neuropathien in prospektiven Studien an großen, multizentrisch rekrutierten und gut definierten Patientensubgruppen validieren lassen.

Bei der multifokal motorischen Neuropathie (MMN) wurden in einer Studie an mehreren, insbesondere auch klinisch und elektrophysiologisch unauffälligen Stellen Verdickungen des Plexus brachialis, sowie der Armnerven beschrieben [42]. Ähnliche Befunde wurden auch von anderen Autoren berichtet $[43,44]$. Auch wurde festgestellt, dass bei MMN die intra- und inter-neurale CSA Variabilität sehr hoch ist, was bei der multifokalen Natur dieser Immunneuropathie nicht überrascht [32]. In einer weiteren Studie wurden 8 MMN Patienten untersucht und es wurden multifokale CSA-Vergrößerungen und auch eine Kaliberzunahme des C6-Spinalnerven im Vergleich mit Kontrollen beschrieben [45]. Einige Fallberichte beschreiben Ultraschallbefunde bei der multifokalen erworbenen demyelinisierenden sensiblen und motorischen Neuropathie (multifocal aqcuired demyelinating sensory and motor neuropathy, MADSAM). Es wird dabei relativ einheitlich der Nachweis von CSA-Vergrößerungen an Segmenten mit elektrophysiologisch nachgewiesenem Leitungsblock beschrieben [46-50]. In $\triangle$ Abb. $\mathbf{3}$ ist das Bei-
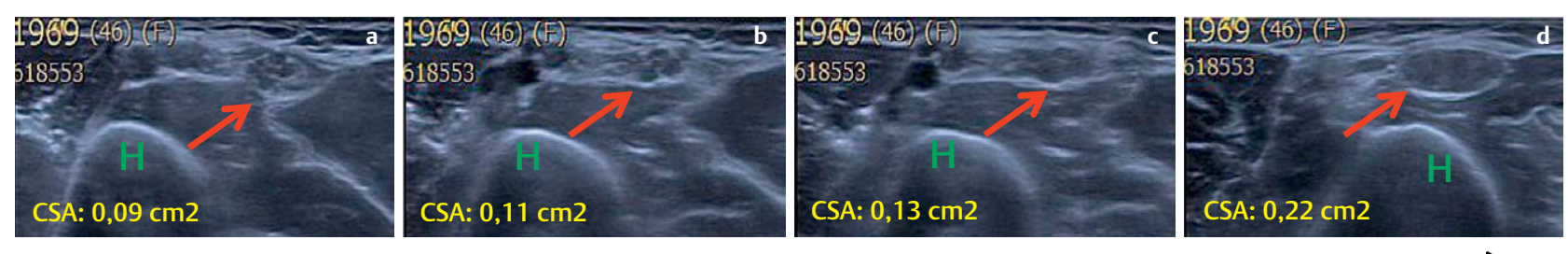

N. ulnaris von distalem nach proximalem Oberarm

Abb. 3 Die Bilderserie a-d zeigt 4 Aufnahmen, die den N. ulnaris (roter Pfeil) in seinem Verlauf vom distalen zum proximalen Oberarm darstellen. Es handelt sich um eine Patientin mit multifokaler erworbener demyelinisierender sensibler und motorischer Neuropathie (MADSAM). In a ist das Nervenkaliber noch verhältnismäßig normal; die Nervenfaszikel sind bereits maulbeerartig vergröbert. In b zeigen sich die Faszikel noch weiter vergrößert, während in c die Abgrenzung der Faszikel im bereits deutlich verdickten Nerven kaum mehr gelingt. In d erscheint der Nerv dann homogen echoarm und stark vergrößert; er hat seine faszikuläre Struktur durch massive ödematöse Schwellung verloren. Das grüne „H“ kennzeichnet den Humerus. CSA: cross sectional area. 
spiel einer Patientin mit MADSAM gezeigt, bei der sich am proximalen Oberarm sonografisch an der Stelle des Leitungsblocks eine massive Schwellung des N. ulnaris fand mit hier praktisch homogen echoarmem Nervenquerschnitt ohne abgrenzbare Faszikel ( $\bullet$ Abb. 3).

Die vaskulitische Neuropathie kann im Sinne eines Organbefalls bei einer systemischen Vaskulitis dabei sein oder sich als nichtsystemische Vaskulitis des peripheren Nervensystems (NSVN) manifestieren [51]. Laut Fallberichten kann man Verdickungen der peripheren Nerven und des Plexus brachialis finden [52] sowie eine Hypoechogenität betroffener Nerven [53]. In einer Studie wurden 14 Patienten mit vaskulitischer Neuropathie im Vergleich zu gesunden Kontrollen untersucht und bei mehreren peripheren Nerven, die auch klinisch und/oder elektrophysiologisch betroffen waren, überwiegend fokale CSA-Verdickungen gefunden [54]. In einer rezent veröffentlichten Studie mit kleiner Fallzahl von Patienten mit histologisch gesicherter NSVN wurde eine Zunahme insbesondere des Längsdurchmessers des N. peronaeus superficialis beschrieben [55]. Systematische Studien mit großen Fallzahlen fehlen.

- Tab. 1 fasst die Befunde bei Immunneuropathien zusammen.

\section{Diabetische Neuropathie}

Auch für die in westlichen Ländern häufigste Art der PNP, die diabetische PNP, liegen Beschreibungen der Sonomorphologie vor. In 2 Studien wurden CSA-Vergrößerungen an den oberen und unteren Extremitäten mittels hochauflösendem Nervenultraschall gezeigt, die z.T. sogar mit den elektrophysiologischen Befunden korrelierten [56,57]. In beiden Studien erfolgte die Untersuchung des N. medianus am Karpaltunnel und somit an einer natürlichen Engpassstelle. Unter Einsatz einer $22 \mathrm{MHz}$ Sonde wurde von der Verdickung des N. suralis bei Patienten mit diabetischer Neuropathie berichtet [58], was allerdings in einer späteren Studie, in der eine $18 \mathrm{MHz}$ Sonde verwendet wurde, nicht bestätigt werden konnte [59]. Kürzlich wurden in einer japanischen Kohorte von Patienten mit Typ II Diabetes im Vergleich mit gesunden Kontrollen mittels Nervenultraschall bereits vor Eintreten einer Neuropathie CSA-Vergrößerungen im $\mathrm{N}$. medianus und N. tibialis posterior nachgewiesen, und zwar außerhalb von Engpassstellen [60]. Falls sich dieser Befund an großen Patientenkohorten bestätigen lässt, wäre er hinsichtlich einer möglichen Früherkennungsfunktion des Nervenultraschalls von großer Relevanz. Zudem wären prospektive Studien, in denen die differenzialdiagnostische Wertigkeit des Nervenultraschalls bei Patienten mit diabetischer Neuropathie im Vergleich mit immunvermittelten Neuropathien wichtig; der Vergleich der Messergebnisse mit Werten bei Immunneuropathien, soweit bei unterschiedlichen Messtechniken und auch verschiedenen Ultraschallgeräten möglich, zeigt bei Diabetes [60] eher geringere Zunahmen der Nervenquerschnittsflächen.

\section{Lepra-assoziierte Neuropathie}

In mehreren Studien wurden Patienten mit Lepra-assoziierter Neuropathie mittels Nervenultraschall untersucht. Bei auch hier uneinheitlicher Datenlage wurde insbesondere von einer N. ulnaris Hypertrophie mit auch deutlicher Verdickung des Perinerium berichtet mit begleitender Hypoechogenität des Nerven [61-63]. Zudem wurde wiederholt eine Hypervaskularisation peripherer Nerven bei Lepra-assoziierter Neuropathie gefunden [64]. In diesem Zusammenhang ist die Beobachtung, dass die beschriebenen Auffälligkeiten nicht auf klinisch und elektrophysiologisch betroffene Nerven beschränkt waren, sondern sonografisch ein viel weiterer Befall des peripheren Nervensystems nachgewiesen werden konnte, bedeutsam. Dabei scheint insbesondere ein asymmetrischer Nervenbefall sonomorphologisch typisch für eine Lepra-assoziierte Neuropathie zu sein [65].

\section{Neuropathie bei amyotropher Lateralsklerose}

Eine wichtige Differenzialdiagnose insbesondere zu rein motorischen PNP wie etwa die MMN ist die amyotrophe Lateralsklerose (ALS). Hier ist die Erweiterung der diagnostischen Möglichkeiten insbesondere mittels nicht-invasiver Verfahren besonders wichtig. Die bislang veröffentlichten Daten deuten darauf hin, dass es bei Patienten mit ALS im Vergleich zu PNP zu einer Reduktion der CSA kommt. Auch wenn die Datenlage mit bislang nur sehr wenigen Studien mit Untersuchungen der Armnerven und zervikalen Nervenwurzeln der wenigen Patienten spärlich ist [66-68], scheinen die Studienergebnisse, die bei diesen Patienten durchweg eine Atrophie des peripheren Nervensystems zeigen, verhältnismäßig einheitlich zu sein. In einer aktuellen Studie konnte gezeigt

Tab. 1 Studien mittels hochauflösendem Ultraschall zu Immunneuropathien.

\begin{tabular}{|c|c|c|}
\hline PNP & Referenzen & Hauptbefunde \\
\hline HNPP & [11-18] & - CSA-Vergrößerungen überwiegend an Engpassstellen \\
\hline $\begin{array}{l}\text { CMT (CMT1A, } \\
\text { CMT1B, CMT2, } \\
\text { CMTX) }\end{array}$ & {$[15,18-24]$} & $\begin{array}{l}\text { - bei demyelinisierenden CMT, generalisierte CSA-Vergrößerung ohne wesentliche Faszikelschwellung } \\
\text { - bei axonalen CMT, Faszikelvergrößerung ohne wesentliche Nervenschwellung }\end{array}$ \\
\hline GBS & {$[5,20,26-28]$} & $\begin{array}{l}\text { - Frühe Schwellung insbesondere proximaler Nervenabschnitte (Nervenwurzeln) } \\
\text { - Fokale CSA-Vergrößerungen und ödematöse Schwellungen der Nervenfaszikel } \\
\text { - CSA-Vergrößerung des N. vagus }\end{array}$ \\
\hline CIDP & {$[7,20,29-40]$} & $\begin{array}{l}\text { - Fokale CSA-Vergrößerung und Faszikelschwellung } \\
\text { - Uneinheitliche Datenlage }\end{array}$ \\
\hline MMN & {$[32,42-45]$} & $\begin{array}{l}\text { - Multifokale CSA-Vergrößerungen an Armnerven, Plexus brachialis, Spinalnerven } \\
\text { - Hohe intra- und inter-neurale CSA-Variabilität }\end{array}$ \\
\hline MADSAM & [46-50] & - Multifokale CSA-Vergrößerungen \\
\hline $\begin{array}{l}\text { Vaskulitische } \\
\text { Neuropathie }\end{array}$ & [52-55] & $\begin{array}{l}\text { - Fokale CSA-Vergrößerungen proximaler und distaler Nervenabschnitte } \\
\text { - Uneinheitliche Datenlage }\end{array}$ \\
\hline
\end{tabular}


werden, dass der Nervenultraschall bei Erfassung von mehreren definierten Messpunkten, wie etwa der CSA mehrerer motorischer Nerven an den oberen und unteren Extremitäten, eine sehr viel höhere diagnostische Treffsicherheit hinsichtlich der Differenzialdiagnose ALS mit überwiegendem Befall des zweiten Motoneurons vs. MMN erreicht als die Elektroneurografie. Zudem fanden sich auch in dieser Studie bei Patienten mit ALS geringere CSA als bei Patienten mit MMN $[45,50]$. In einer aktuellen Studie wurde berichtet, dass bei ALS Patienten die Untersuchung zervikaler Nervenwurzeln mittels hochauflösendem Nervenultraschall keinen relevanten Kaliberunterschied zu gesunden Kontrollen zeigt [69]. Desweiteren sollte bei der Frage nach einer ALS auch der Muskelultraschall ergänzend zum Nervenultraschall zum Einsatz kommen [70,71]. Bei höherer Sensitivität gelingt insbesondere der Nachweis von Faszikulationen und das Ausmaß an Muskelatrophie damit sehr viel schneller als etwa mit der Elektromyographie.

\section{Hochauflösender Ultraschall bei PNP - die Einschränkungen \\ $\nabla$}

Auch wenn der Einsatz von hochauflösendem Nervenultraschall bei der Diagnostik von PNP in den letzten Jahren eine sehr positive Entwicklung genommen hat, sind die Probleme und Einschränkungen ebenfalls offensichtlich. Die größte Herausforderung bei der Anwendung des Nervenultraschalls und beim Datenvergleich ist die auf mehreren Ebenen fehlende Standardisierung. Mangels nationaler und internationaler Leitlinien gibt es bspw. kein einheitliches Vorgehen dazu, welche Nerven bzw. Nervenkombinationen und welche Nervenabschnitte bei definierten Fragestellungen untersucht werden sollten. Es fehlt Konsensus darüber, an welchen Landmarken quantitative Messungen vorzunehmen sind, welche Messungen genau durchgeführt werden sollten, bei welchen Geräteeinstellungen diese Messungen zu erfolgen haben, und wie sie zu dokumentieren sind $[25,72,73]$. Diese Aspekte sind nicht nur beim klinischen Einsatz des Nervenultraschalls wichtig, sondern insbesondere auch dann, wenn es um den Vergleich von Daten aus unterschiedlichen wissenschaftlichen Studien geht [74]. Weiterhin zu berücksichtigen ist der Einsatz verschiedener Ultraschallgeräte mit unterschiedlich leistungsstarken Schallsonden, sowie die jeweils benutzte Software. All diese Aspekte können dazu beitragen, die zahlreichen vermeintlich diskrepanten Ergebnisse in der derzeitigen Literatur zum Thema hochauflösender Nervenultraschall bei PNP zu erklären. Hinzu kommen die Anwendung unterschiedlicher Diagnosekriterien bei der Patientenrekrutierung sowie die oft fehlende Mituntersuchung einer gesunden Kontrollpopulation bei zudem häufig sehr niedrigen Fallzahlen. Die Reproduktion der Messergebnisse sowie die verblindete Datenauswertung sind weitere wesentliche Aspekte, die im Sinne der Vergleichbarkeit und Datenqualität in künftigen Studien dringend berücksichtigt werden müssen. Wie bei jeder Untersuchungstechnik spielt auch beim Nervenultraschall die Untersuchererfahrung eine entscheidende Rolle. Regelmäßige Übung und eine curriculare Ausbildung (http://www.degum.de/ fileadmin/dokumente/sektionen/neurologie/richtlinien/Ausbil dungskurrikulumMNUSVers2010.pdf) sichern die Basis dieser grundsätzlich einfachen Untersuchungsmethode - für die klinische Anwendung wie auch für wissenschaftliche Studien. Es ist wichtig die eigenen Grenzen und auch die Grenzen des eigenen Ultraschallgerätes zu kennen, um erhobene Befunde korrekt ein- ordnen und werten zu können. Wie auch bei elektrophysiologischen Messungen ist zu empfehlen, eigene Labor-interne Normwerte zu generieren, mit denen die bei Patienten erhobenen Werte verglichen werden können; die potenziellen Einflussfaktoren auf Messergebnisse sind zu mannigfaltig, als dass Werte aus unterschiedlichen Labors unkritisch übernommen werden könnten.

\section{Hochauflösender Ultraschall bei PNP - die Chancen $\nabla$}

Der hochauflösende Nervenultraschall alleine kann im Falle von PNP momentan noch keine Diagnose stellen. Er gestattet aber zusätzlich zu den klinischen und elektrophysiologischen Befunden die Erweiterung der diagnostischen Möglichkeiten um eine sehr wertvolle weitere Dimension. Der Nervenultraschall ist eine begeisternde Methode, die nicht nur dem Patienten wegen der nicht-invasiven und schmerzfreien Untersuchung sehr angenehm ist, sondern auch dem Untersucher bereits nach sehr kurzer Zeit sehr viel Spaß machen wird. Sie ist derzeit die einzige Untersuchungstechnik, die die dynamische Darstellung der anatomischen Verhältnisse im peripheren Nervensystem erlaubt, und zwar in Längs- und Querschnitt. Die Untersuchungsmethode gibt einen und erreicht problemlos auch Nervenabschnitte wie Plexus und Nervenwurzeln, die elektroneurografisch praktisch nicht zugänglich sind. Nervenultraschall ist daher auch gut für Verlaufsuntersuchungen nach eingeleiteter Therapie geeignet. Der Nervenultraschall kann bei PNP differenzialdiagnostischwertvolle Hinweise geben, wenn es z.B. um die Frage nach entzündlicher vs. hereditärer PNP geht, um die Differenzierung eines Nerven- vs. Wurzelschadens, oder etwa darum, eine PNP von einer ALS zu unterscheiden. Desweiteren können insbesondere bei Mononeuropathien die oft nicht bedachten nicht-neurologischen Ursachen wie etwa Raumforderungen aus dem umgebenden Gewebe erkannt und entsprechender (chirurgischer) Therapie zugeführt werden ( $\bullet$ Abb. 4). Ein weiteres Einsatzgebiet ist die Erkennung von geeigneten Entnahmestellen für eine Nerven- oder Faszikelbiopsie ( $\bullet$ Abb. 5). In beiden Fällen erleichtert der Nervenultraschall die präoperative Planung, hilft die operative Komplikationsrate zu reduzieren und die diagnostische Treffsicherheit der entnommenen Biopsate zu erhöhen.

\section{Ausblick \\ $\nabla$}

Die hochauflösende Ultraschalldiagnostik wird in den nächsten Jahren weiter in die PNP-Diagnostik Einzug halten - sowohl bei der Differenzialdiagnostik, als auch bei der Ortung geeigneter Nerven- bzw. Faszikelbiopsiestellen. Bereits die aktuell verfügbaren $12-20 \mathrm{MHz}$ Sonden liefern exzellente Bilder, auf denen man die Struktur und die Umgebung der peripheren Nerven, der Nervenplexus und der Nervenwurzen beurteilen kann. Dies wird durch die ständige Weiterentwicklung der Ultraschallgeräte und ihrer Software künftig noch mehr verbessert werden. Die Ultraschalldiagnostik krankt derzeit noch an der mangelnden Standardisierung. Hier sind in den nächsten Jahren nationale und internationale Leitlinien notwendig, um in der klinischen Routine, aber auch bei wissenschaftlichen Arbeiten die Aussagekraft zu maximieren, während die Fehlerrate reduziert wird. Entscheidend ist in diesem Zusammenhang auch die Festlegung von Untersuchungsprogrammen, die die zu untersuchenden Nerven und durchzuführenden Messungen an definierten 


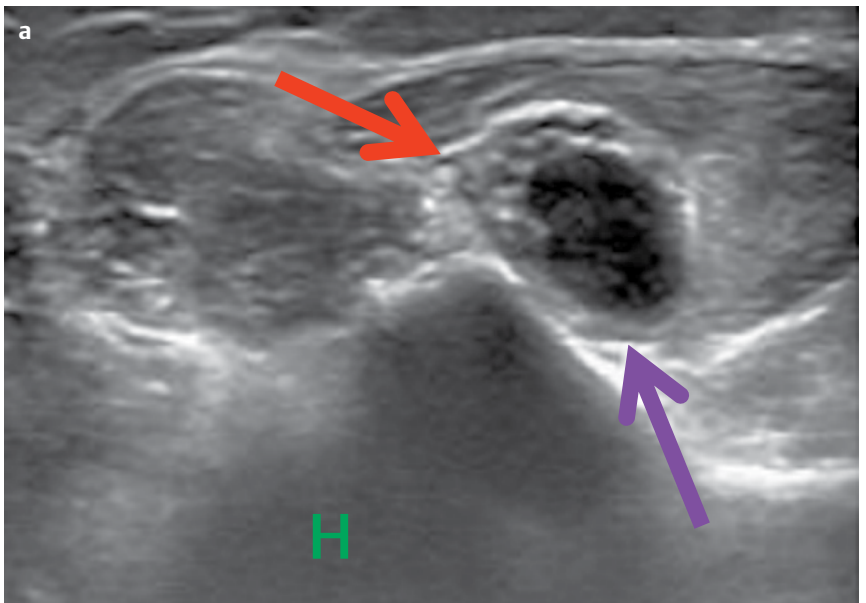

Abb. 4 Dargestellt ist der N. ulnaris (a, roter Pfeil), der durch eine ca. $1 \mathrm{~cm}$ große, homogen echoarme Raumforderung (violetter Pfeil) wenige $\mathrm{cm}$ distal des Sulcus ulnaris so stark bedrängt wird, dass nur noch ein sichelförmiger schmaler Nervensaum am linken Oberpol der Raumforderung zu erkennen ist (a, roter Pfeil). Wenige $\mathrm{mm}$ weiter proximal $\mathbf{b}$, aber immer noch distal des Sulcus ulnaris erreicht die Raumforderung (violetter Pfeil) ihr Größenmaximum; hier ist der Nerv so stark komprimiert, dass er praktisch nicht mehr

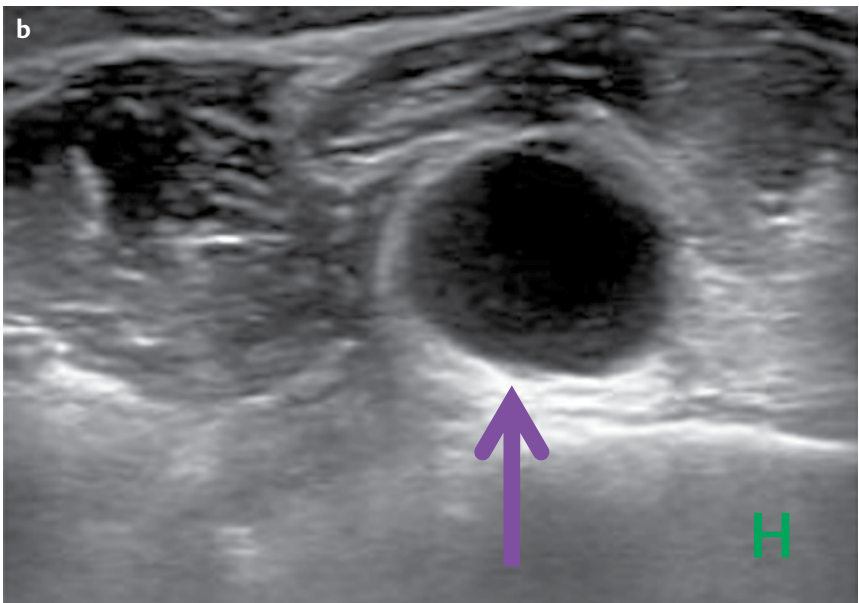

abgegrenzt werden kann. Der Patient beklagte akut aufgetretene massive lokale Dauerschmerzen knapp unterhalb des Sulcus ulnaris und bot ausgeprägte Paresen der N. ulnaris-versorgten Muskulatur zusammen mit einem Taubheitsgefühl im Versorgungsgebiet des N. ulnaris. Nach neurochirurgischer Entfernung ergab die histopathologische Begutachtung den Befund eines Sehnenscheidenganglions.

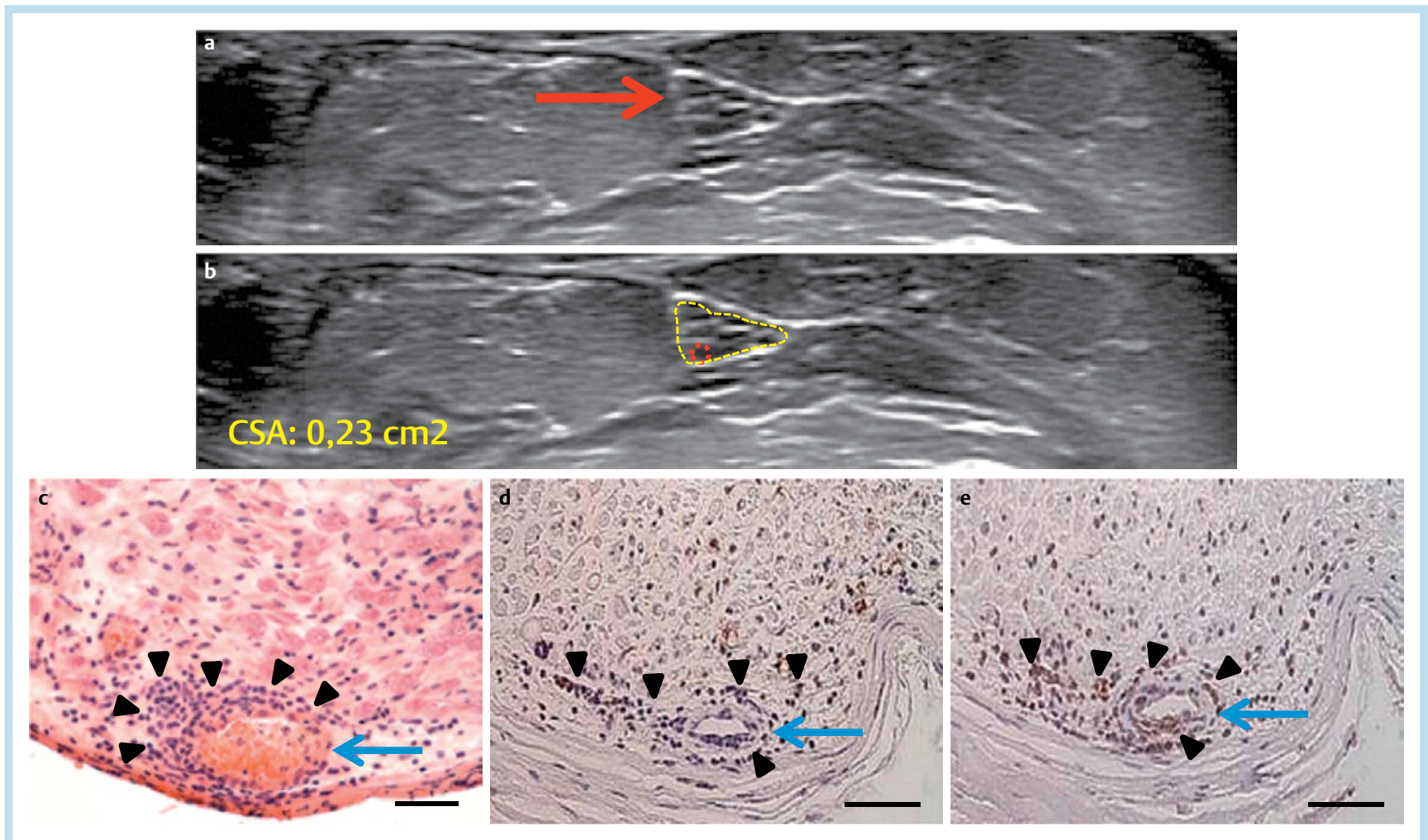

Abb. 5 Mittels hochauflösendem Ultraschall detektierte maulbeerartige Auftreibung des N. ulnaris in Unterarmmitte (a, roter Pfeil; b, gelbe gestrichelte Markierung), aus dem eine Faszikelbiopsie entnommen wurde (b, rote gestrichelte Markierung innerhalb der gelben gestrichelten Markierung). Die histologische Aufarbeitung des Faszikelbiopsats zeigte in der Haemalaun-Eosin-Färbung ein dichtes peri- und transmurales Zellinfiltrat (c, schwarze Pfeilköpfe) an einem endoneuralen Gefäß (c, blauer Pfeil). In diesem Infiltrat konnten sowohl T-Zellen (d, schwarze Pfeilköpfe) als auch Makrophagen (e, schwarze Pfeilköpfe) nachgewiesen werden. Es wurde die Diagnose einer vaskulitischen Polyneuropathie gestellt, die mit immunsuppressiver Therapie behandelt werden konnte. Balken in c: $50 \mu \mathrm{m}$; Balken in d und e: $250 \mu \mathrm{m}$. CSA: cross sectional area.
Messorten vorgeben. Zudem ist die strukturierte Ausbildung der Sonografeure/-eusen nach einem Curriculum notwendig, um die Untersuchungsqualität zu steigern. Prospektive, multi-zentrische Studien an großen und gut definierten PNP-Patientengruppen im Vergleich zu gesunden Kontrollpersonen mit gut strukturierten und standardisierten Studienprotokollen, kontrollierter Datenerhebung und verblindeter Datenauswertung durch gut ausgebildete Untersucher und Ausschaltung möglicher externer und interner Einflussfaktoren werden in den 
nächsten Jahren helfen den hochauflösenden Nervenultraschall noch besser in der Diagnostik von PNP zu verankern.

\section{Interessenkonflikt}

Die Autorin gibt an, dass kein Interessenkonflikt besteht.

\section{Literatur}

1 Kollmer J, Bendszus M, Pham M. Mr. neurography: diagnostic imaging in the PNS. Clin Neuroradiol 2015; 25 (Suppl 2): 283-289

2 Chhabra A, Flammang A, Padua A Jr. et al. Magnetic resonance neurography: technical considerations. Neuroimaging Clin N Am 2014; 24: 67-78

3 Bartels RH, Meulstee J, Verhagen WI et al. Ultrasound imaging of the ulnar nerve: correlation of preoperative and intraoperative dimensions. Clin Neurol Neurosurg 2008; 110: 687-690

4 Goedee HS, Brekelmans GJ, van Asseldonk JT et al. High resolution sonography in the evaluation of the peripheral nervous system in polyneuropathy - a review of the literature. Eur J Neurol 2013; 20: 1342-1351

5 Grimm A, Decard BF, Axer H. Ultrasonography of the peripheral nervous system in the early stage of Guillain-Barre syndrome. J Peripher Nerv Syst 2014; 19: 234-241

6 Grimm A, Heiling B, Schumacher $U$ et al. Ultrasound differentiation of axonal and demyelinating neuropathies. Muscle Nerve 2014; 50: 976-983

7 Kerasnoudis A, Pitarokoili K, Behrendt $V$ et al. Correlation of nerve ultrasound, electrophysiological and clinical findings in chronic inflammatory demyelinating polyneuropathy. J Neuroimaging 2015; 25 : 207-216

8 Mhoon JT, Juel VC, Hobson-Webb LD. Median nerve ultrasound as a screening tool in carpal tunnel syndrome: correlation of cross-sectional area measures with electrodiagnostic abnormality. Muscle Nerve 2012; 46: 871-878

9 Hobson-Webb LD, Massey JM, Juel VC et al. The ultrasonographic wristto-forearm median nerve area ratio in carpal tunnel syndrome. Clin Neurophysiol 2008; 119: 1353-1357

10 Cartwright MS, Walker FO. Neuromuscular ultrasound in common entrapment neuropathies. Muscle Nerve 2013; 48: 696-704

11 Tekin L, Özgül A. Ultrasound aids in the diagnosis of hereditary neuropathy with liability to pressure palsies. Surg Neurol 2009; 71: 399-400, discussion 400

12 Beekman R, Visser LH. Sonographic detection of diffuse peripheral nerve enlargement in hereditary neuropathy with liability to pressure palsies. J Clin Ultrasound 2002; 30: 433-436

$13 \mathrm{Kim}$ SH, Yang SN, Yoon JS et al. Sonographic evaluation of the peripheral nerves in hereditary neuropathy with liability to pressure palsies: a case report. Ann Rehabil Med 2014; 38: 109-115

14 Hooper DR, Lawson W, Smith L et al. Sonographic features in hereditary neuropathy with liability to pressure palsies. Muscle Nerve 2011; 44: 862-867

15 Schreiber S, Oldag A, Kornblum C et al. Sonography of the median nerve in CMT1A, CMT2A, CMTX, and HNPP. Muscle Nerve 2013; 47: 385-395

16 Bayrak AO, Bayrak IK, Battaloğlu E et al. Ultrasonographic findings in hereditary neuropathy with liability to pressure palsies. Neurol Res 2015; 37: 106-111

17 Lucchetta M, Dalla Torre C, Granata G et al. Sonographic features in hereditary neuropathy with liability to pressure palsies. Muscle Nerve 2012; 45: 920-921; author reply 921-922

18 Goedee SH, Brekelmans GJ, van den Berg LH et al. Distinctive patterns of sonographic nerve enlargement in Charcot-Marie-Tooth type $1 \mathrm{~A}$ and hereditary neuropathy with pressure palsies. Clin Neurophysiol 2015; 126: 1413-1420

19 Cartwright MS, Brown ME, Eulitt P et al. Diagnostic nerve ultrasound in Charcot-Marie-Tooth disease type 1B. Muscle Nerve 2009; 40: 98-102

20 Zaidman CM, Al-Lozi M, Pestronk A. Peripheral nerve size in normals and patients with polyneuropathy: an ultrasound study. Muscle Nerve 2009; 40: 960-966

21 Heinemeyer 0 , Reimers $C D$. Ultrasound of radial, ulnar, median, and sciatic nerves in healthy subjects and patients with hereditary motor and sensory neuropathies. Ultrasound Med Biol 1999; 25: 481-485

22 Martinoli C, Schenone A, Bianchi $S$ et al. Sonography of the median nerve in Charcot-Marie-Tooth disease. AJR Am J Roentgenol 2002; 178: $1553-1556$
23 Noto Y, Shiga K, Tsuji Y et al. Nerve ultrasound depicts peripheral nerve enlargement in patients with genetically distinct Charcot-MarieTooth disease. J Neurol Neurosurg Psychiatry 2015; 86: 378-384

24 Grimm A, Rasenack M, Athanasopoulou IM et al. The modified ultrasound pattern sum score mUPSS as additional diagnostic tool for genetically distinct hereditary neuropathies. J Neurol 2016; 263: 221-230

25 Padua L, Briani C. The different ultrasound patterns in Charcot-MarieTooths raise the need of standardization. Clin Neurophysiol 2015; 126: 1286-1287

26 Almeida V, Mariotti $P$, Veltri $S$ et al. Nerve ultrasound follow-up in a child with Guillain-Barre syndrome. Muscle Nerve 2012; 46: 270-275

27 Gallardo E, Sedano MJ, Orizaola $P$ et al. Spinal nerve involvement in early Guillain-Barre syndrome: a clinico-electrophysiological, ultrasonographic and pathological study. Clin Neurophysiol 2015; 126: 810-819

28 Grimm A, Decard BF, Schramm A et al. Ultrasound and electrophysiologic findings in patients with Guillain-Barre syndrome at disease onset and over a period of six months. Clin Neurophysiol 2016; 127: 1657-1663

29 Granata G, Pazzaglia C, Calandro P et al. Ultrasound visualization of nerve morphological alteration at the site of conduction block. Muscle Nerve 2009; 40: 1068-1070

30 Taniguchi $N$, Itoh $K$, Wang $Y$ et al. Sonographic detection of diffuse peripheral nerve hypertrophy in chronic inflammatory demyelinating polyradiculoneuropathy. J Clin Ultrasound 2000; 28: 488-491

31 Imamura $K$, Tajiri $Y$, Kowa $H$ et al. Peripheral nerve hypertrophy in chronic inflammatory demyelinating polyradiculoneuropathy detected by ultrasonography. Intern Med 2009; 48: 581-582

32 Padua L, Martinoli C, Pazzaglia C et al. Intra- and internerve crosssectional area variability: new ultrasound measures. Muscle Nerve 2012; 45: 730-733

33 Matsuoka N, Kohriyama T, Ochi K et al. Detection of cervical nerve root hypertrophy by ultrasonography in chronic inflammatory demyelinating polyradiculoneuropathy. J Neurol Sci 2004; 219: 15-21

34 Rajabally YA, Morlese J, Kathuria D et al. Median nerve ultrasonography in distinguishing neuropathy sub-types: a pilot study. Acta Neurol Scand 2012; 125: 254-259

35 Zaidman CM, Pestronk $A$. Nerve size in chronic inflammatory demyelinating neuropathy varies with disease activity and therapy response over time: a retrospective ultrasound study. Muscle Nerve 2014; 50: 733-738

36 Jang JH, Cho CS, Yang KS et al. Pattern analysis of nerve enlargement using ultrasonography in chronic inflammatory demyelinating polyneuropathy. Clin Neurophysiol 2014; 125: 1893-1899

37 Di Pasquale A, Morino S, Loreti S et al. Peripheral nerve ultrasound changes in CIDP and correlations with nerve conduction velocity. Neurology 2015; 84: 803-809

38 Grimm A, Vittore D, Schubert $V$ et al. Ultrasound aspects in therapynaive CIDP compared to long-term treated CIDP. J Neurol 2016, doi: 10.1007/s00415-016-8100-9

39 Padua L, Granata G, Sabatelli $M$ et al. Heterogeneity of root and nerve ultrasound pattern in CIDP patients. Clin Neurophysiol 2014; 125: 160-165

40 Kerasnoudis A, Pitarokoili K, Behrendt $V$ et al. Nerve ultrasound score in distinguishing chronic from acute inflammatory demyelinating polyneuropathy. Clin Neurophysiol 2014; 125: 635-641

41 Kerasnoudis A, Pitarokoili K, Gold $R$ et al. Bochum ultrasound score allows distinction of chronic inflammatory from multifocal acquired demyelinating polyneuropathies. J Neurol Sci 2015; 348: 211-215

42 Beekman $R$, van den Berg LH, Franssen $H$ et al. Ultrasonography shows extensive nerve enlargements in multifocal motor neuropathy. Neurology 2005; 65: 305-307

43 Pitarokoili K, Gold R, Yoon MS. Nerve ultrasound in a case of multifocal motor neuropathy without conduction block. Muscle Nerve 2015; 52 : 294-299

44 Kerasnoudis A. Correlation of sonographic and electrophysiological findings in a patient with multifocal motor neuropathy. J Neuroimaging 2014; 24: 305-307

45 Grimm A, Decard BF, Athanasopoulou I et al. Nerve ultrasound for differentiation between amyotrophic lateral sclerosis and multifocal motor neuropathy. J Neurol 2015; 262: 870-880

46 Scheidl E, Bohm J, Simo $M$ et al. Ultrasonography of MADSAM neuropathy: focal nerve enlargements at sites of existing and resolved conduction blocks. Neuromuscul Disord 2012; 22: 627-631

47 Simon NG, Kiernan MC. Precise correlation between structural and electrophysiological disturbances in MADSAM neuropathy. Neuromuscul Disord 2015; 25: 904-907 
48 Neubauer C, Gruber H, Bauerle J et al. Ultrasonography of multifocal acquired demyelinating sensory and motor neuropathy (MADSAM). Clin Neuroradiol 2015; 25: 423-425

49 Grimm A, Rasenack M, Athanasopoulou I et al. Long-term observations in asymmetric immune-mediated neuropathy with vagus hypertrophy using ultrasound of the nerves. J Neurol Sci 2015; 356: 205-208

50 Loewenbruck KF, Liesenberg J, Dittrich $M$ et al. Nerve ultrasound in the differentiation of multifocal motor neuropathy (MMN) and amyotrophic lateral sclerosis with predominant lower motor neuron disease (ALS/LMND). J Neurol 2016; 263: 35-44

51 Collins MP, Periquet MI, Mendell JR et al. Nonsystemic vasculitic neuropathy: insights from a clinical cohort. Neurology 2003; 61: 623-630

52 Nodera $H$, Sato K, Terasawa Y et al. High-resolution sonography detects inflammatory changes in vasculitic neuropathy. Muscle Nerve 2006; 34: $380-381$

53 Ito $T$, Kijima $M$, Watanabe $T$ et al. Ultrasonography of the tibial nerve in vasculitic neuropathy. Muscle Nerve 2007; 35: 379-382

54 Grimm A, Decard BF, Bischof A et al. Ultrasound of the peripheral nerves in systemic vasculitic neuropathies. J Neurol Sci 2014; 347: 44-49

55 Üçeyler N, Schäfer K, Mackenrodt D et al. High-resolution ultrasonography of the superficial peroneal 1 motor and sural sensory nerves may be a non-invasive approach 2 to the diagnosis of vasculitic neuropathy. Front Neurol 2016; 7: 48

56 Watanabe T, Ito H, Sekine A et al. Sonographic evaluation of the peripheral nerve in diabetic patients: the relationship between nerve conduction studies, echo intensity, and cross-sectional area. J Ultrasound Med 2010; 29: 697-708

57 Watanabe T, Ito $H$, Morita $A$ et al. Sonographic evaluation of the median nerve in diabetic patients: comparison with nerve conduction studies. J Ultrasound Med 2009; 28: 727-734

58 Liu F, Zhu J, Wei M et al. Preliminary evaluation of the sural nerve using 22-MHz ultrasound: a new approach for evaluation of diabetic cutaneous neuropathy. PLoS One 2012; 7: e32730

59 Hobson-Webb LD, Massey JM, Juel VC. Nerve ultrasound in diabetic polyneuropathy: correlation with clinical characteristics and electrodiagnostic testing. Muscle Nerve 2013; 47: 379-384

60 Ishibashi F, Taniguchi M, Kojima R et al. Morphological changes of the peripheral nerves evaluated by high-resolution ultrasonography are associated with the severity of diabetic neuropathy, but not corneal nerve fiber pathology in patients with type 2 diabetes. J Diabetes Investig 2015 ; 6 : 334-342
61 Martinoli C, Derchi LE, Bertolotto $M$ et al. US and MR imaging of peripheral nerves in leprosy. Skeletal Radiol 2000; 29: 142-150

62 Elias J Jr., Nogueira-Barbosa MH, Feltrin LT et al. Role of ulnar nerve sonography in leprosy neuropathy with electrophysiologic correlation. J Ultrasound Med 2009; 28: 1201-1209

63 Bathala L, Kumar K, Pathapati $R$ et al. Ulnar neuropathy in hansen disease: clinical, high-resolution ultrasound and electrophysiologic correlations. J Clin Neurophysiol 2012; 29: 190-193

64 Jain S, Visser LH, Praveen TL et al. High-resolution sonography: a new technique to detect nerve damage in leprosy. PLoS Negl Trop Dis 2009; 3: e498

65 Lugao HB, Nogueira-Barbosa MH, Marques W Jr. et al. Asymmetric nerve enlargement: a characteristic of leprosy neuropathy demonstrated by ultrasonography. PLoS Negl Trop Dis 2015; 9: e0004276

66 Schreiber S, Abdulla S, Debska-Vielhaber G et al. Peripheral nerve ultrasound in amyotrophic lateral sclerosis phenotypes. Muscle Nerve 2015; 51: 669-675

67 Nodera H, Takamatsu N, Shimatani $Y$ et al. Thinning of cervical nerve roots and peripheral nerves in ALS as measured by sonography. Clin Neurophysiol 2014; 125: 1906-1911

68 Cartwright MS, Walker FO, Griffin LP et al. Peripheral nerve and muscle ultrasound in amyotrophic lateral sclerosis. Muscle Nerve 2011; 44: 346-351

69 Mori A, Nodera H, Takamatsu $N$ et al. Sonographic evaluation of cervical nerve roots in ALS and its clinical subtypes. J Med Invest 2016; 63: $54-57$

70 Arts IM, Overeem S, Pillen S et al. Muscle ultrasonography: a diagnostic tool for amyotrophic lateral sclerosis. Clin Neurophysiol 2012; 123: 1662-1667

71 Grimm A, Prell T, Decard BF et al. Muscle ultrasonography as an additional diagnostic tool for the diagnosis of amyotrophic lateral sclerosis. Clin Neurophysiol 2015; 126: 820-827

72 Hobson-Webb $L D$, Boon AJ. Reporting the results of diagnostic neuromuscular ultrasound: an educational report. Muscle Nerve 2013; 47: 608-610

73 Jelsing EJ, Presley JC, Maida E et al. The effect of magnification on sonographically measured nerve cross-sectional area. Muscle Nerve 2015; 51: 30-34

74 Boehm J, Scheidl E, Bereczki $D$ et al. High-resolution ultrasonography of peripheral nerves: measurements on 14 nerve segments in 56 healthy subjects and reliability assessments. Ultraschall Med 2014; 35: $459-467$ 